\title{
Erratum to: Characterization of a novel stilbene synthase promoter involved in pathogen- and stress-inducible expression from Chinese wild Vitis pseudoreticulata
}

\author{
Weirong Xu - Yihe $\mathrm{Yu} \cdot$ Jiahua Ding $\cdot$ Zhanyong Hua \\ Yuejin Wang
}

Published online: 15 December 2009

(C) Springer-Verlag 2009

\section{Erratum to: Planta}

\section{DOI 10.1007/s00425-009-1062-8}

Unfortunately, in Fig. 1, labels ("EF1 $\gamma$ " and " $\beta$-Glu") were missing in the online published article.

The online version of the original article can be found under doi:10.1007/s00425-009-1062-8.

W. Xu $\cdot$ Y. Yu $\cdot$ J. Ding $\cdot$ Z. Hua $\cdot$ Y. Wang $(\bowtie)$

Key Laboratory of Horticulture Plant Germplasm, Utilization in Northwest China, Ministry of Agriculture,

712100 Yangling, Shaanxi, China

e-mail: wangyj@nwsuaf.edu.cn

W. Xu

e-mail: xwr9320@163.com

W. Xu $\cdot$ Y. Yu $\cdot$ J. Ding $\cdot$ Z. Hua $\cdot$ Y. Wang College of Horticulture, Northwest A\&F University, 712100 Yangling, Shaanxi, China

W. Xu $\cdot$ Y. Yu $\cdot$ J. Ding $\cdot$ Z. Hua $\cdot$ Y. Wang Key Laboratory of Agricultural Molecular Biology of Shaanxi Province, Northwest A\&F University, 712100 Yangling, Shaanxi, China
The correct figure is displayed below.

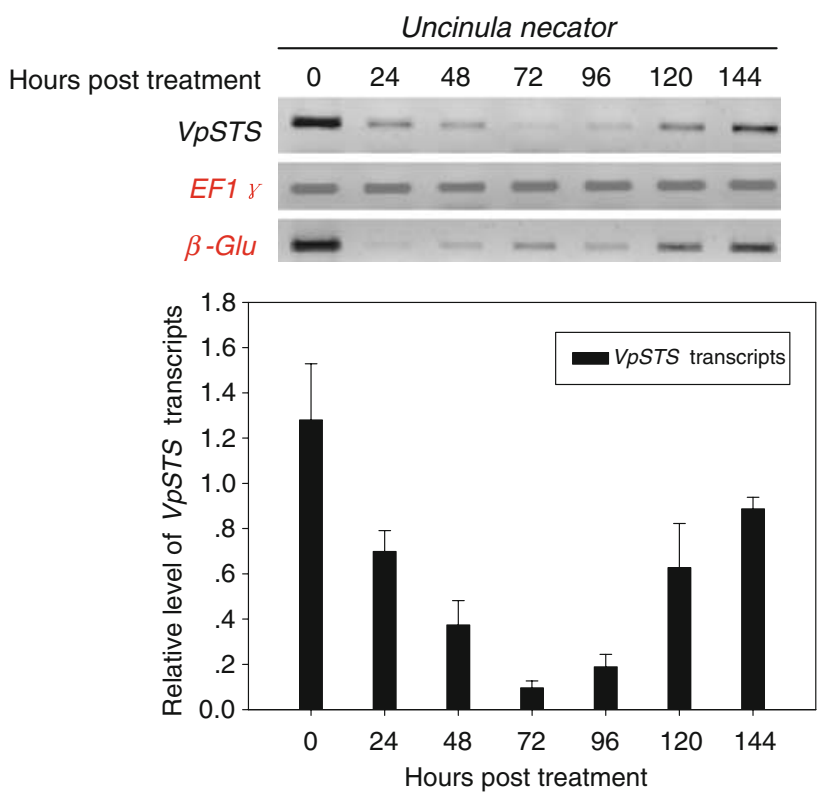

Fig. 1 VpSTS expression in response to Uncinula necator. Leaves were harvested at the indicated times after Uncinula necator inoculation for preparation of total RNA. Accumulation of VpSTS and $\beta$-Glu ( $\beta-1,3$ glucanase gene) transcripts was monitored by semiquantitative RT-PCR. EF1 $\gamma$ amplification was used as constitutive control. VpSTS transcript accumulation was quantified by densitometry using Bio imaging Systems software (Syngene) for two independent biological experiments $(n=2)$; each value was corrected according to the $\mathrm{EF} 1 \gamma$ amplification signal and expressed as a ratio between the corrected value and the control time point (at $0 \mathrm{~h}$ ) corrected value. Error bars indicate standard error of means 